\title{
RELACIÓN ENTRE PERIMETRÍA AUTOMATIZADA CONVENCIONAL Y HRT, OCT Y GDX EN SUJETOS NORMALES, HIPERTENSOS OCULARES Y GLAUCOMATOSOS
}

\author{
RELATIONSHIP BETWEEN STANDARD AUTOMATED \\ PERIMETRY AND HRT, OCT AND GDX IN NORMAL, OCULAR \\ HYPERTESIVE AND GLAUCOMATOUS SUBJECTS
}

\author{
LÓPEZ-PEÑA MJ ${ }^{1}$, FERRERAS A ${ }^{2}$, POLO V², LARROSA JM ${ }^{2}$, HONRUBIA FM $^{2}$
}

\section{RESUMEN}

Objetivo: Determinar las correlaciones entre los parámetros estructurales de la cabeza del nervio óptico y CFNR obtenidos mediante la topografía papilar (HRT II), la tomografía óptica de coherencia (Stratus OCT 3000) y la polarimetría láser (GDx VCC), con los índices globales de la perimetría automatizada convencional en sujetos normales, hipertensos oculares y con glaucoma.

Material y método: Trescientos ochenta y nueve ojos fueron incluidos en el estudio y clasificados en 3 grupos: 43 normales, 274 hipertensos oculares y 72 glaucomas según los resultados de la presión intraocular basal y la PA. Se calcularon las correlaciones de Pearson entre los índices principales de la PA, desviación media (DM) y desviación estándar de la media (DSM), y los parámetros morfométricos papilares y de la capa de fibras nerviosas de la retina (CFNR) obtenidos con el HRT II, OCT y GDx VCC, en los diferentes grupos diagnósticos.

\section{ABSTRACT}

Purpose: To determine the correlations between structural parameters of the optic nerve head and the retinal nerve fiber layer (RNFL) obtained by using a Heidelberg Retina Tomograph II (HRT II), optic coherence tomography (OCT), and laser polarimetry (GDX-VCC) and the perimetric indices of standard automated perimetry (SAP) in normal, ocular hypertensive and glaucomatous subjects.

Methods: Three hundred and eighty-nine patients were enrolled in the study and classified into three separate groups: 43 with normal eyes, 274 with ocular hypertensive eyes and 72 with glaucomatous eyes. Subjects were classified according to the basal intraocular pressure and the SAP results. Pearson's correlation coefficients were calculated between the global perimetric indices, mean deviation (MD) and pattern standard deviation (PSD), and structural parameters of the RNFL and optic disc obtained by using HRT II, OCT and GDX-VCC in the different diagnostic groups.

\footnotetext{
Recibido: 23/3/06. Aceptado: 26/3/07.

Hospital Universitario Miguel Servet. Zaragoza. España.

1 Licenciado en Medicina.

2 Doctor en Medicina.

Correspondencia:

María Jesús López Peña

Hospital Universitario Miguel Servet

Servicio de Oftalmología

C/. Isabel la Católica, 1-3

50009 Zaragoza

España

E-mail: mariaj.lopez@yahoo.com
} 
Resultados: La mayoría de los parámetros estructurales del HRT, OCT y GDx presentaron correlaciones débiles o moderadas con los índices principales de la PA, en el grupo de glaucomas. Los normales e hipertensos mostraron correlaciones débiles o no significativas. Las correlaciones fueron más fuertes para la DM que para la DSM. Los parámetros basados en el estudio de la CFNR presentaron correlaciones más fuertes que los que se basaron en el estudio de la cabeza del nervio óptico.

Conclusiones: La concordancia entre defectos del campo visual y alteraciones de la cabeza del nervio óptico o CFNR es débil, lo que justifica la interpretación conjunta de la información aportada por las pruebas estructurales y funcionales para evaluar con mayor precisión el daño glaucomatoso.

Palabras clave: Glaucoma, HRT, OCT, GDX VCC, campo visual.

\section{INTRODUCCIÓN}

El glaucoma crónico simple es una neuropatía óptica en la que se producen cambios morfológicos en la cabeza del nervio óptico y capa de fibras nerviosas de la retina (CFNR), así como alteraciones funcionales consistentes en una pérdida progresiva e irreversible del campo visual.

En los últimos años se han desarrollado varias técnicas de análisis estructural por imagen, que aportan datos cuantitativos reproducibles que eliminan la subjetividad y variabilidad interobservador.

Numerosos trabajos (1-5) han mostrado la capacidad de la topografía papilar con el láser confocal de barrido Heidelberg Retina Tomograph (HRT), del protocolo de análisis para el glaucoma de la tomografía óptica de coherencia (OCT) y de la polarimetría láser (GDx) para detectar alteraciones glaucomatosas en la papila o CFNR. Aunque algunos de ellos no encuentran diferencias significativas entre dichos instrumentos y la evaluación cualitativa de un oftalmólogo experimentado, todos ellos coinciden en su capacidad para diferenciar sujetos normales de glaucomatosos y en el aumento del poder discriminante al combinarlos.

La valoración funcional del daño glaucomatoso es básica en el estudio del glaucoma. No hay unanimidad a la hora de establecer si se puede detectar primero el daño estructural o las pérdidas del campo visual, pero en la valoración conjunta de ambos,
Results: Mild to moderate correlations were found between the structural parameters measured by HRT, OCT and GDX and the global perimetric indices, in the glaucoma group. Mild or no significant correlations were found in the normal and ocular hypertensive groups. Correlations were stronger for MD than for PSD. Parameters based on the study of the retinal nerve fiber layer showed stronger correlations than those based on the study of the optic nerve head.

Conclusions: The relationship between structural and functional measurements in glaucoma is weak and therefore the results of these ancillary tests should be interpreted together to increase diagnostic accuracy (Arch Soc Esp Oftalmol 2007; 82: 197 208).

Key words: Glaucoma, HRT, OCT, GDX-VCC, visual field.

se basa la práctica clínica del diagnóstico del glaucoma.

El objetivo de este estudio es comparar los parámetros morfométricos obtenidos mediante el HRT, el OCT y el GDX con los índices globales: desviación media (DM) y desviación estándar de la media (DSM) de la perimetría automatizada convencional (PA), en una población de sujetos divididos en normales, hipertensos oculares y glaucomatosos.

\section{SUJETOS, MATERIAL Y MÉTODOS}

\section{Sujetos}

Se pre-incluyeron un total de 400 sujetos seleccionados de forma prospectiva y consecutiva a partir de las consultas de oftalmología de nuestro centro. Dos de ellos no firmaron el consentimiento informado, seis no completaron todas las pruebas incluidas en el protocolo exploratorio y en tres fue imposible obtener unos resultados fiables en alguna de las pruebas realizadas. Finalmente, 389 ojos fueron incluidos en el estudio siempre y cuando reunieran los criterios de inclusión descritos a continuación: Edad entre 30 y 75 años, agudeza visual mayor o igual a 20/30 (escala de Snellen), defectos de refracción menores de 5 dioptrías esféricas y astigmatismo menor de 3 dioptrías de equivalente 
esférico y medios ópticos transparentes (color $\mathrm{u}$ opalescencia nuclear, cortical o subcapsular posterior del cristalino $<1$ según el Lens Opacities Classification System III) (6).

Se excluyeron aquellos sujetos con antecedentes de traumatismos, cirugía ocular previa, enfermedades sistémicas con repercusión oftálmica o incapacidad para la realización de alguna de las pruebas incluidas en el protocolo de estudio.

A todos se les realizó una exploración oftalmológica completa; biomicroscopía con lámpara de hendidura, gonioscopía, medición de los niveles de presión intraocular (PIO) mediante tonometría de aplanación, paquimetría corneal central (DGH Technology, modelo DGH 500), fondo de ojo mediante oftalmoscopía indirecta, estereofotografías papilares, perimetría automatizada convencional (PA), topografía papilar con el Heidelberg Retina Tomograph (HRT), evaluación de los espesores de la capa de fibras de la retina mediante la tomografía óptica de coherencia (OCT) y una polarimetría láser con el GDx con compensación corneal variable (GDx VCC).

Se solicitó consentimiento informado y se siguieron las directrices de la declaración de Helsinki. Sólo se incluyó un ojo de cada paciente en el estudio. Si ambos eran elegibles se seleccionó uno de forma aleatoria. Así se obtuvieron 3 grupos:

Sujetos normales: PIO menor de $21 \mathrm{~mm}$ de $\mathrm{Hg}$ y ausencia de defectos en la PA. Hipertensos oculares (HTO): $\mathrm{PIO} \geq$ a $21 \mathrm{~mm}$ de $\mathrm{Hg}$ (en al menos tres mediciones en días diferentes) y PA normal. Sujetos glaucomatosos: $\mathrm{PIO}>$ a $21 \mathrm{~mm}$ de $\mathrm{Hg}$, y defectos glaucomatosos en la PA, con independencia de la morfología papilar (tabla I).

\section{Perimetría automatizada convencional}

La PA se realizó mediante un perímetro Humphrey Field Analyzer modelo 745 (estrategia 24-2 SITA standard). Los defectos perimétricos fueron definidos siguiendo los criterios de Caprioli (7) y/o considerando como índices globales: DM o DSM con un nivel de probabilidad menor al $2 \%$; y/o prueba del hemicampo glaucomatoso fuera de límites normales. Se realizaron al menos dos perimetrías para disminuir el efecto aprendizaje, y si alguna no cumplía los criterios de validez definidos por el propio perímetro, se repitió.

\section{Láser confocal de barrido: HRT II}

La exploración con HRT II fue llevada a cabo por un único examinador experimentado (JML). Para su realización se dilataron las pupilas con tropicamida al $1 \%$.

Se obtuvieron 3 series de imágenes a partir de las cuales se elaboró una única que fue usada para el análisis de datos. El mismo experto en planimetría (que desconocía cualquier dato clínico del paciente), delimitó el contorno de la papila. El software del HRT II (Heidelberg Eye Explorer versión 1.4.1.0) establece automáticamente un plano de referencia y calcula parámetros globales (los únicos considerados en este estudio) y sectoriales de la cabeza del nervio óptico (tablas II-IV). El análisis de datos del HRT II también incluye los resultados de la regresión de Moorfields (8) clasificando a los pacientes en 3 grupos: normal (dentro del $95 \%$ de la población normal), límite (entre el 95 y el 99,9\%) y

Tabla I. Características clínicas de los grupos del estudio

\begin{tabular}{|c|c|c|c|c|c|c|c|c|c|c|c|}
\hline & \multicolumn{2}{|c|}{ Total } & \multicolumn{3}{|c|}{ Normales } & \multicolumn{3}{|c|}{ HTO } & \multicolumn{3}{|c|}{ Glaucomas } \\
\hline & Media & DS & Media & DS & $\mathrm{P}$ & Media & DS & $\mathrm{p}$ & Media & DS & $\mathrm{P}$ \\
\hline DM & $-1,55$ & 3,71 & $-1,28$ & 2,39 & G & $-0,30$ & 1,12 & G & $-6,54$ & 6,03 & $\mathrm{~N}, \mathrm{H}$ \\
\hline DSM & 1,77 & 2,35 & 1,47 & 1,62 & G & 0,97 & 0,75 & G & 5,06 & 3,66 & $\mathrm{~N}, \mathrm{H}$ \\
\hline Edad & 58,38 & 11,74 & 58,95 & 11,14 & & 56,10 & 11,63 & & 61,62 & 9,36 & \\
\hline AV & 0,90 & 0,12 & 0,89 & 0,10 & G & 0,92 & 0,11 & G & 0,83 & 0,13 & $\mathrm{~N}, \mathrm{H}$ \\
\hline $\mathrm{PIO}$ & 22,03 & 4,43 & 14,40 & 2,37 & $\mathrm{H}, \mathrm{G}$ & 23,03 & 3,04 & $\mathrm{~N}, \mathrm{G}$ & 24,31 & 3,89 & $\mathrm{~N}, \mathrm{H}$ \\
\hline Área disco & 2,13 & 0,38 & 2,08 & 0,36 & & 2,19 & 0,43 & & 2,16 & 0,38 & \\
\hline E/D vertical & 0,56 & 0,23 & 0,47 & 0,27 & G & 0,54 & 0,20 & G & 0,73 & 0,18 & $\mathrm{~N}, \mathrm{H}$ \\
\hline Paquimetría & 556,08 & 37,63 & 552,78 & 30,18 & & 557,53 & 37,86 & & 543,74 & 36,04 & \\
\hline
\end{tabular}

Total

389

43

274

72

N: Diferencias con el grupo normal; H: Diferencias con el grupo de HTO; G: Diferencias con el grupo de glaucomas; DE: desviación estándar; DM: desviación media; DSPC: desviación estándar patrón corregida; AV: agudeza visual; PIO: presión intraocular. 
Tabla II. Correlación de los índices principales de la PA con los parámetros morfométricos papilares del HRT II en el grupo control

\begin{tabular}{lcccccc}
\hline Correlaciones de Pearson & $\mathrm{DM}$ & $\mathrm{p}$ & $\mathrm{IC} 95 \%$ & $\mathrm{DSM}$ & $\mathrm{p}$ & $\mathrm{IC} 95 \%$ \\
\hline Área disco & 0,111 & 0,478 & $-0,1958 / 0,3982$ & $-0,268$ & 0,082 & $-0,5262 / 0,0349$ \\
Área excavación & 0,251 & 0,104 & $-0,0530 / 0,5129$ & $-0,299$ & 0,052 & $-0,5498 / 0,0017$ \\
Área anillo & $-0,175$ & 0,262 & $-0,4516 / 0,1323$ & 0,053 & 0,737 & $-0,2515 / 0,3477$ \\
Relación área exc/disco & $\mathbf{0 , 3 0 4}$ & $\mathbf{0 , 0 4 8}$ & $\mathbf{0 . 0 0 3 6 / 0 , 5 5 3 5}$ & $\mathbf{- 0 , 3 1 5}$ & $\mathbf{0 , 0 4 0}$ & $\mathbf{- 0 , 5 6 2 0 / - 0 , 0 1 5 9}$ \\
Relación área anillo/disco & $\mathbf{- 0 , 3 0 4}$ & $\mathbf{0 , 0 4 8}$ & $\mathbf{- 0 , 5 5 3 5 / - 0 , 0 0 3 6}$ & $\mathbf{0 , 3 1 5}$ & $\mathbf{0 , 0 4 0}$ & $\mathbf{0 , 0 1 5 9 / 0 , 5 6 2 0}$ \\
Vol exc & 0,172 & 0,271 & $-0,1357 / 0,4488$ & $-0,187$ & 0,229 & $-0,4618 / 0,1196$ \\
Vol anillo & $-0,126$ & 0,420 & $-0,4110 / 0,1810$ & 0,055 & 0,725 & $-0,2493 / 0,3497$ \\
Prof media exc & 0,168 & 0,281 & $-0,1391 / 0,4461$ & $-0,191$ & 0,219 & $-0,4650 / 0,1156$ \\
Máx prof exc & 0,180 & 0,247 & $-0,1269 / 0,4560$ & $-0,163$ & 0,296 & $-0,4417 / 0,1444$ \\
Variación alt contorno & $-0,025$ & 0,873 & $-0,3230 / 0,2774$ & 0,092 & 0,557 & $-0,2143 / 0,3818$ \\
Morf exc & $-0,150$ & 0,337 & $-04310 / 0,1573$ & $-0,036$ & 0,818 & $-0,3328 / 0,2672$ \\
Espesor medio CFNR & $-0,140$ & 0,371 & $-0,4224 / 0,1675$ & 0,092 & 0,559 & $-0,2145 / 0,3816$ \\
Área de sección CFNR & $-0,107$ & 0,496 & $-0,3943 / 0,2001$ & $-0,003$ & 0,985 & $-0,3030 / 0,2977$ \\
Relación exc/disco hor & $\mathbf{0 , 3 4 5}$ & $\mathbf{0 , 0 2 3}$ & $\mathbf{0 , 0 5 0 1 / 0 , 5 8 4 9}$ & $\mathbf{- 0 , 3 3 7}$ & $\mathbf{0 , 0 2 7}$ & $\mathbf{- 0 , 5 7 8 5 / - 0 , 0 4 0 3}$ \\
Relación exc/disco vert & $\mathbf{0 , 3 0 5}$ & $\mathbf{0 , 0 4 7}$ & $\mathbf{0 , 0 0 5 3 / 0 , 5 5 4 7}$ & $\mathbf{- 0 , 3 4 4}$ & $\mathbf{0 , 0 2 4}$ & $\mathbf{- 0 , 5 8 4 1 / - 0 , 0 4 8 8}$ \\
Elev máx contorno & $-0,060$ & 0,702 & $-0,3541 / 0,2446$ & $-0,089$ & 0,570 & $-0,3794 / 0,2170$ \\
Depre máx contorno & $-0,058$ & 0,710 & $-0,3525 / 0,2464$ & 0,003 & 0,985 & $-0,2977 / 0,3030$ \\
CLM TS & $-0,016$ & 0,917 & $-0,3152 / 0,2853$ & $-0,001$ & 0,997 & $-0,3008 / 0,2999$ \\
CLM TI & 0,117 & 0,454 & $-0,1897 / 0,4035$ & $-0,045$ & 0,776 & $-0,3405 / 0,2591$ \\
Variabilidad media & $-0,286$ & 0,063 & $-0,5399 / 0,0157$ & 0,145 & 0,354 & $-0,1627 / 0,4266$ \\
Altura de referencia & $-0,097$ & 0,535 & $-0,3864 / 0,2091$ & 0,007 & 0,962 & $-0,2935 / 0,3071$ \\
FSM & $-0,047$ & 0,766 & $-0,3423 / 0,2572$ & 0,042 & 0,789 & $-0,2617 / 0,3381$ \\
RB & 0,103 & 0,512 & $-0,2038 / 0,3911$ & 0,068 & 0,663 & $-0,2368 / 0,3613$ \\
\hline \hline
\end{tabular}

DM: desviación media; DSM: desviación estándar de la media; Vol. Exc: volumen excavación.

Tabla III. Correlación de los índices principales de la PA con los parámetros morfométricos papilares del HRT II en el grupo de HTO

\begin{tabular}{lcccccc}
\hline Correlaciones de Pearson & DM & $\mathrm{p}$ & $\mathrm{IC} 95 \%$ & $\mathrm{DSM}$ & $\mathrm{p}$ & $\mathrm{IC} 95 \%$ \\
\hline Área disco & 0,033 & 0,577 & $-0,0700 / 0,1953$ & $-0,058$ & 0,332 & $-0,1768 / 0,0890$ \\
Área excavación & 0,002 & 0,972 & $-0,0111 / 0,2514$ & $-0,043$ & 0,469 & $-0,2080 / 0,0568$ \\
Área anillo & 0,047 & 0,425 & $-0,1877 / 0,0779$ & $-0,026$ & 0,660 & $-0,1028 / 0,1633$ \\
Relación área exc/disco & $-0,014$ & 0,814 & $-0,0198 / 0,2432$ & $-0,014$ & 0,819 & $-0,1864 / 0,0792$ \\
Relación área anillo/disco & 0,014 & 0,814 & $-0,2432 / 0,0198$ & 0,014 & 0,819 & $-0,0792 / 0,1864$ \\
Vol exc & 0,000 & 0,998 & $-0,0050 / 0,2571$ & $-0,030$ & 0,614 & $-0,2212 / 0,0430$ \\
Vol anillo & $-0,007$ & 0,911 & $-0,2511 / 0,0114$ & $-0,009$ & 0,884 & $-0,1047 / 0,1614$ \\
Prof media exc & 0,011 & 0,849 & $-0,0593 / 0,2056$ & 0,001 & 0,983 & $-0,1861 / 0,0795$ \\
Máx prof exc & $-0,055$ & 0,355 & $-0,1399 / / 0,1265$ & 0,004 & 0,940 & $-0,1863 / 0,0792$ \\
Variación alt contorno & $-0,108$ & 0,068 & $-0,2585 / 0,0035$ & 0,092 & 0,119 & $-0,0927 / 0,1732$ \\
Morf exc & 0,044 & 0,457 & $0,0011 / 0,2627$ & 0,019 & 0,753 & $-0,1272 / 0,1392$ \\
Espesor medio CFNR & $-0,122$ & 0,072 & $-0,2513 / 0,0111$ & $-0,002$ & 0,975 & $-0,1175 / 0,1488$ \\
Área de sección CFNR & 0,041 & 0,490 & $-0,2196 / 0,0447$ & $-0,020$ & 0,740 & $-0,1330 / 0,1334$ \\
Relación exc/disco hor & $-0,038$ & 0,518 & $-0,0981 / 0,1679$ & $-0,045$ & 0,444 & $-0,2209 / 0,0434$ \\
Relación exc/disco vert & $-0,016$ & 0,785 & $-0,0621 / 0,2030$ & 0,010 & 0,864 & $-0,1507 / 0,1155$ \\
Elev máx contorno & 0,094 & 0,113 & $-0,0259 / 0,2374$ & $-0,082$ & 0,166 & $-0,2276 / 0,0363$ \\
Depre máx contorno & $-0,037$ & 0,530 & $-0,1768 / 0,0890$ & 0,030 & 0,609 & $-0,1643 / 0,1018$ \\
CLM TS & $-0,093$ & 0,117 & $-0,2250 / 0,0390$ & 0,081 & 0,171 & $-0,1057 / 0,1604$ \\
CLM TI & 0,066 & 0,266 & $-0,2774 /-0,0169$ & $-0,086$ & 0,146 & $-0,1665 / 0,0995$ \\
Variabilidad media & $\mathbf{- 0 , 1 8 5}$ & $\mathbf{0 , 0 0 2}$ & $\mathbf{- 0 , 2 9 3 8 / - 0 , 0 3 4 8}$ & 0,053 & 0,369 & $-0,0817 / 0,1840$ \\
Altura de referencia & 0,034 & 0,563 & $-0,1661 / 0,1000$ & $-0,016$ & 0,793 & $-0,1766 / 0,0893$ \\
FSM & $-0,130$ & 0,054 & $-0,2594 / 0,0025$ & $-0,028$ & 0,639 & $-0,0907 / 0,1752$ \\
RB & $-0,040$ & 0,496 & $-0,3314 /-0,0763$ & $-0,028$ & 0,641 & $-0,1429 / 0,1234$ \\
\hline \hline
\end{tabular}

DM: desviación media; DSM: desviación estándar de la media; Vol. Exc: volumen excavación. 
Tabla IV. Correlación de los índices principales de la PA con los parámetros morfométricos papilares del HRT II en el grupo de glaucomas

\begin{tabular}{lcccccc}
\hline Correlaciones de Pearson & $\mathrm{DM}$ & $\mathrm{p}$ & $\mathrm{IC} 95 \%$ & $\mathrm{DSM}$ & $\mathrm{p}$ & $\mathrm{IC} 95 \%$ \\
\hline Área disco & $-0,106$ & 0,370 & $-0,3263 /-0,1259$ & 0,043 & 0,715 & $-0,1872 / 0,2690$ \\
Área excavación & $-0,381$ & 0,001 & $-0,5608 /-0,1672$ & 0,338 & 0,003 & $0,1187 / 0,5260$ \\
Área anillo & 0,423 & 0,000 & $0,2152 / 0,5940$ & $-0,456$ & 0,000 & $-0,6199 /-0,2540$ \\
Relación área exc/disco & $-0,474$ & 0,000 & $-0,6335 /-0,2748$ & 0,455 & 0,000 & $0,2527 / 0,6191$ \\
Relación área anillo/disco & 0,474 & 0,000 & $0,2748 / 0,6335$ & $-0,455$ & 0,000 & $-0,6191 /-0,2527$ \\
Vol exc & $-0,268$ & 0,021 & $-0,4680 /-0,0422$ & 0,319 & 0,006 & $0,0977 / 0,5104$ \\
Vol anillo & 0,291 & 0,012 & $0,0673 / 0,4874$ & $-0,302$ & 0,009 & $-0,4959 /-0,0785$ \\
Prof media exc & $-0,176$ & 0,134 & $-0,3886 / 0,0550$ & 0,258 & 0,027 & $0,0311 / 0,4592$ \\
Máx prof exc & $-0,155$ & 0,187 & $-0,3703 / 0,0763$ & 0,181 & 0,123 & $-0,0498 / 0,3931$ \\
Variación alt contorno & $-0,129$ & 0,275 & $-0,3469 / 0,1029$ & 0,221 & 0,058 & $-0,0075 / 0,4283$ \\
Morf exc & $-0,290$ & 0,012 & $-0,4860 /-0,0655$ & 0,299 & 0,010 & $0,0761 / 0,4941$ \\
Espesor medio CFNR & 0,375 & 0,001 & $0,1605 / 0,5561$ & $-0,298$ & 0,010 & $-0,4929 /-0,0745$ \\
Área de sección CFNR & 0,348 & 0,002 & $0,1298 / 0,5340$ & $-0,282$ & 0,015 & $-0,4794 /-0,0570$ \\
Relación exc/disco hor & $-0,298$ & 0,010 & $-0,4929 /-0,0746$ & 0,278 & 0,016 & $0,0529 / 0,4763$ \\
Relación exc/disco vert & $-0,437$ & 0,000 & $-0,6054 /-0,2321$ & 0,387 & 0,001 & $0,1743 / 0,5658$ \\
Elev máx contorno & $-0,062$ & 0,601 & $-0,2862 / 0,1692$ & $-0,075$ & 0,525 & $-0,2984 / 0,1562$ \\
Depre máx contorno & $-0,174$ & 0,137 & $-0,3874 / 0,0564$ & 0,193 & 0,099 & $-0,0367 / 0,4041$ \\
CLM TS & 0,353 & 0,002 & $0,1351 / 0,5378$ & $-0,087$ & 0,462 & $-0,3092 / 0,1446$ \\
CLM TI & 0,241 & 0,038 & $0,0136 / 0,4453$ & $-0,396$ & 0,000 & $-0,5729 /-0,1844$ \\
Variabilidad media & $-0,195$ & 0,096 & $-0,4053 / 0,0352$ & 0,145 & 0,219 & $-0,0868 / 0,3612$ \\
Altura de referencia & 0,140 & 0,233 & $-0,0910 / 0,3574$ & $-0,155$ & 0,188 & $-0,3702 / 0,0764$ \\
FSM & 0,338 & 0,003 & $0,1183 / 0,5257$ & $-0,434$ & 0,000 & $-0,6025 /-0,2277$ \\
RB & 0,423 & 0,000 & $0,2149 / 0,5938$ & $-0,419$ & 0,000 & $-0,5907 /-0,2103$ \\
\hline \hline
\end{tabular}

DM: desviación media; DSM: desviación estándar de la media; Vol. Exc: volumen excavación.

fuera de límites normales (por encima del 99,9\% de la población normal). Se consideraron imágenes de buena calidad aquellas con una desviación estándar entre barridos menor de 30 a.m.

\section{Tomografía óptica de coherencia}

Realizada mediante el Stratus OCT 3000 de manera estandarizada por un único examinador (JML) tras instilar tropicamida al 1\%. Se realizaron protocolos de estudio correspondientes a la CFNR y papila. Las medidas utilizadas para el análisis de los datos fueron obtenidas de la media de los 3 barridos realizados en el primer protocolo obteniendo datos de espesor (global, por cuadrantes y sectores) y parámetros morfométricos papilares a partir de 6 barridos radiales a nivel del disco óptico (área de disco, área de excavación, área de anillo, relación área excavación-disco, relación horizontal excavación-disco y relación vertical excavación-disco). Se consideraron imágenes de buena calidad aquellas con una relación señal/ruido mayor de $30 \mathrm{~dB}$ o más de un $95 \%$ de barridos A aceptados.

\section{Polarimetría láser (GDX VCC)}

El estudio con GDX VCC fue realizado bajo midriasis farmacológica (tropicamida 1\%). El aparato realiza una compensación de la birrefringencia del segmento anterior de forma individualizada para cada ojo y calcula el espesor de la CFNR en el área peripapilar en función de la luz reflejada en la retina. Los cálculos se realizan sobre una banda de tamaño fijo centrada en el nervio óptico, obteniendo parámetros morfológicos: Promedio superior, inferior, desviación TSNIT y mapas o imágenes del área estudiada. Además aporta un índice que es mayor cuanto mayor es la probabilidad de glaucoma: el NFI (neve fiber indicator). La simetría interojo no fue valorada puesto que sólo se utilizó un ojo de cada sujeto.

\section{Análisis estadístico}

Para establecer si había diferencias estadísticas $(\mathrm{p}<0,05)$ en las características clínicas se calcularon las ANOVAs entre los diferentes subgrupos (com- 
Tabla V. Correlación de los índices principales del campo visual con los parámetros de fibras y papila de la OCT en el grupo control

\begin{tabular}{|c|c|c|c|c|c|c|}
\hline Correlaciones de Pearson & DM & $\mathrm{p}$ & IC $95 \%$ & DSM & $\mathrm{p}$ & IC $95 \%$ \\
\hline H1 (sentido horario OD) & $-0,052$ & 0,439 & $-0,4845 / 0,0907$ & 0,259 & 0,093 & $-0,0445 / 0,5192$ \\
\hline H2 (horas OD) & 0,165 & 0,290 & $-0,1424 / 0,4434$ & $-0,164$ & 0,293 & $-0,4427 / 0,1432$ \\
\hline H3 (horas OD) & 0,098 & 0,532 & $-0,2084 / 0,3870$ & $-0,104$ & 0,506 & $-0,3923 / 0,2025$ \\
\hline H4 (horas OD) & $-0,125$ & 0,425 & $-0,4098 / 0,1824$ & 0,154 & 0,324 & $-0,4098 / 0,1824$ \\
\hline H5 (horas OD) & $-0,174$ & 0,264 & $-0,4510 / 0,1331$ & 0,116 & 0,460 & $-0,1913 / 0,4021$ \\
\hline H6 (horas OD) & $-0,064$ & 0,684 & $-0,3573 / 0,2412$ & $-0,008$ & 0,958 & $-0,3078 / 0,2928$ \\
\hline H7 (horas OD) & 0,292 & 0,057 & $-0,0092 / 0,5446$ & $-0,304$ & 0,047 & $-0,5539 /-0,0041$ \\
\hline H8 (horas OD) & 0,087 & 0,580 & $-0,2192 / 0,3774$ & $-0,054$ & 0,732 & $-0,3486 / 0,2506$ \\
\hline H9 (horas OD) & 0,180 & 0,248 & $-0,1270 / 0,4559$ & $-0,134$ & 0,392 & $-0,4173 / 0,1736$ \\
\hline H10 (horas OD) & 0,203 & 0,192 & $-0,1037 / 0,4744$ & $-0,145$ & 0,355 & $-0,4264 / 0,1628$ \\
\hline H11 (horas OD) & 0,245 & 0,113 & $-0,0595 / 0,5082$ & $-0,211$ & 0,174 & $-0,4809 / 0,0954$ \\
\hline H12 (horas OD) & $-0,050$ & 0,463 & $-0,5370 / 0,0199$ & 0,252 & 0,103 & $-0,0523 / 0,5135$ \\
\hline Superior & $-0,205$ & 0,059 & $-0,4238 / 0,1659$ & 0,158 & 0,313 & $-0,1499 / 0,4372$ \\
\hline Inferior & $-0,067$ & 0,322 & $-0,2814 / 0,3191$ & $-0,089$ & 0,572 & $-0,3789 / 0,2175$ \\
\hline Nasal & 0,036 & 0,818 & $-0,2672 / 0,3328$ & $-0,049$ & 0,755 & $-0,3442 / 0,2552$ \\
\hline Temporal & 0,148 & 0,344 & $-0,1597 / 0,4291$ & $-0,133$ & 0,393 & $-0,4171 / 0,1738$ \\
\hline Promedio de la CFNR & $-0,052$ & 0,447 & $-0,3731 / 0,2239$ & 0,043 & 0,786 & $-0,2610 / 0,3387$ \\
\hline Espesor global CFNR & $-0,105$ & 0,502 & $-0,3930 / 0,2016$ & 0,026 & 0,866 & $-0,2761 / 0,3243$ \\
\hline $\operatorname{Imax} / \mathrm{Smax}$ & 0,026 & 0,870 & $-0,2768 / 0,3235$ & $-0,109$ & 0,487 & $-0,3962 / 0,1980$ \\
\hline Smax/Imax & $-0,020$ & 0,897 & $-0,3187 / 0,2817$ & 0,098 & 0,531 & $-0,2083 / 0,3871$ \\
\hline Smax/Tavg & $-0,153$ & 0,328 & $-0,4331 / 0,1548$ & 0,156 & 0,318 & $-0,1516 / 0,4358$ \\
\hline Imax/Tavg & $-0,214$ & 0,168 & $-0,4832 / 0,0924$ & 0,138 & 0,378 & $-0,1694 / 0,4209$ \\
\hline Smax/Navg & $-0,051$ & 0,744 & $-0,3462 / 0,2530$ & 0,072 & 0,645 & $-0,2330 / 0,3648$ \\
\hline Max-Min & $-0,027$ & 0,863 & $-0,3248 / 0,2754$ & $-0,004$ & 0,980 & $-0,3040 / 0,2967$ \\
\hline SMAX & $-0,047$ & 0,486 & $-0,2786 / 0,3217$ & 0,044 & 0,779 & $-0,2597 / 0,3400$ \\
\hline IMAX & $-0,078$ & 0,249 & $-0,2602 / 0,3394$ & $-0,062$ & 0,691 & $-0,3561 / 0,2425$ \\
\hline SAVG & $-0,071$ & 0,297 & $-0,4238 / 0,1659$ & 0,158 & 0,313 & $-0,1499 / 0,4372$ \\
\hline IAVG & 0,068 & 0,313 & $-0,2814 / 0,3191$ & $-0,089$ & 0,572 & $-0,3789 / 0,2175$ \\
\hline Espesor medio CFNR & $-0,101$ & 0,136 & $-0,2822 / 0,3183$ & $-0,023$ & 0,882 & $-0,3213 / 0,2791$ \\
\hline Área vert del anillo & $-0,015$ & 0,925 & $-0,3137 / 0,2869$ & 0,080 & 0,611 & $-0,2260 / 0,3712$ \\
\hline Anchura horiz del anillo & $-0,130$ & 0,056 & $-0,2988 / 0,3078$ & 0,075 & 0,634 & $-0,2307 / 0,3669$ \\
\hline Área de disco & 0,289 & 0,060 & $-0,0123 / 0,5424$ & $-0,314$ & 0,040 & $-0,5617 /-0,0156$ \\
\hline Área de excavación & $\mathbf{0 , 3 2 4}$ & 0,034 & $\mathbf{0 , 0 2 5 8 / 0 , 5 6 8 7}$ & $-0,400$ & $\mathbf{0 , 0 0 8}$ & $-0,6252 /-0,1132$ \\
\hline Área de anillo & $-0,188$ & 0,417 & $-0,3887 / 0,2065$ & 0,161 & 0,302 & $-0,1461 / 0,4403$ \\
\hline Relación de área E/D & $\mathbf{0 , 3 2 0}$ & $\mathbf{0 , 0 3 6}$ & $0,0216 / 0,5659$ & $-0,402$ & $\mathbf{0 , 0 0 8}$ & $-0,6264 /-0,1152$ \\
\hline Relación horizontal E/D & 0,388 & 0,010 & $0,0995 / 0,6167$ & $-0,491$ & 0,001 & $-0,6894 /-0,2231$ \\
\hline Relación vertical E/D & 0,334 & 0,028 & $\mathbf{0 , 0 3 7 9 / 0 , 5 7 6 8}$ & $-0,409$ & $\mathbf{0 , 0 0 6}$ & $-0,6316 /-0,1236$ \\
\hline
\end{tabular}

DM: desviación media; DSM: desviación estándar de la media.

paraciones múltiples post-hoc de Scheffe). Para valorar la bondad de ajuste de las variables evaluadas se aplicó el test de Kolmogorov-Smirnov para una distribución de contrate normal. Para las variables que se ajustaron a la normalidad se calcularon las correlaciones de Pearson entre los índices principales de la PA y los parámetros estructurales del HRT, OCT y GDx en cada grupo diagnóstico. Para las variables que no se ajustaron a una distribución normal se utilizaron las correlaciones no paramétricas de Spearman. Los análisis estadísticos se realizaron con el programa SPSS (v 11.01, SPSS Inc. Chicago, Illinois) y MedCalc (v 8.2. MedCalc Software, Mariakerke, Bélgica).

\section{RESULTADOS}

En el 97,17\% de los casos, se obtuvieron imágenes de buena calidad con el HRT en la primera exploración realizada. Para el OCT esta circunstancia ocurrió en el $94,34 \%$ y en el $92,54 \%$ para el GDx VCC.

La tabla I muestra las características clínicas de los 389 ojos de la población de estudio y de los subgrupos en los que se dividió: 43 normales, 274 hipertensos oculares y 72 glaucomas. Se encontraron diferencias estadísticamente significativas $(\mathrm{p}<0,05 \%)$ en la DM de la PA, la DSM de la PA, la relación excavación disco-vertical valorada 
mediante estereofotografías y la agudeza visual, entre el grupo de glaucomas y los otros dos. La PIO basal media fue diferente en los tres grupos. La paquimetría corneal central, la edad media y el área de disco no presentaron diferencias entre los tres grupos.

El test de Kolmogorov-Smirnov demostró que todas las variables incluidas siguieron una distribución normal, por lo que se calcularon las correlaciones de Pearson. En las tablas II-X las correlaciones significativas $(\mathrm{p}<0,05)$ presentan una coloración más fuerte. Los coeficientes de Pearson más altos aparecen coloreados de rojo. Se calcularon las ecuaciones de regresión para las mejores asociaciones entre la PA y el resto de pruebas realizadas.
Los índices principales de la PA (DM y DSM) presentaron correlaciones débiles o moderadas con algunos parámetros estructurales del HRT II en el grupo de glaucoma (tabla IV y fig. 1). Los normales e hipertensos (tablas II y III) apenas mostraron correlaciones significativas. Las más fuertes (superiores a 0,4) se observaron para: Área de anillo, relación del área excavación/disco y del área ani1lo/disco, relación excavación/disco vertical y función discriminante RB en el grupo de glaucomas.

El OCT mostró la misma tendencia. Por grupos, los normales e hipertensos mostraron pocas correlaciones significativas (tablas V y VI), a diferencia del grupo de glaucomas (tabla VII) donde se observaron las correlaciones más fuertes y en el mayor

Tabla VI. Correlación de los índices principales del campo visual con los parámetros de fibras y papila de la OCT en el grupo de HTO

\begin{tabular}{|c|c|c|c|c|c|c|}
\hline Correlaciones de Pearson & $\mathrm{DM}$ & $\mathrm{p}$ & IC $95 \%$ & DSM & $\mathrm{p}$ & IC $95 \%$ \\
\hline H1 (sentido horario OD) & 0,083 & 0,159 & $-0,1847 / 0,0810$ & $-0,088$ & 0,136 & $-0,1982 / 0,0671$ \\
\hline H2 (horas OD) & 0,055 & 0,355 & $-0,1929 / 0,0725$ & $-0,202$ & 0,001 & $-0,3275 /-0,0719$ \\
\hline H3 (horas OD) & 0,042 & 0,474 & $-0,1515 / 0,1148$ & $-0,104$ & 0,080 & $-0,2776 /-0,0171$ \\
\hline H4 (horas OD) & 0,069 & 0,245 & $-0,1362 / 0,1302$ & $-0,082$ & 0,168 & $-0,2317 / 0,0320$ \\
\hline H5 (horas OD) & 0,079 & 0,183 & $-0,1501 / 0,1162$ & $-0,101$ & 0,089 & $-0,2455 / 0,0174$ \\
\hline H6 (horas OD) & 0,039 & 0,507 & $-0,2182 / 0,0462$ & $-0,077$ & 0,191 & $-0,2498 / 0,0128$ \\
\hline H7 (horas OD) & 0,092 & 0,119 & $-0,1725 / 0,0934$ & $-0,097$ & 0,102 & $-0,2349 / 0,0286$ \\
\hline H8 (horas OD) & 0,014 & 0,815 & $-0,1972 / 0,0681$ & $-0,012$ & 0,842 & $-0,1606 / 0,1056$ \\
\hline H9 (horas OD) & $-0,066$ & 0,264 & $-0,2371 / 0,0262$ & 0,013 & 0,826 & $-0,1674 / 0,0986$ \\
\hline H10 (horas OD) & $-0,058$ & 0,326 & $-0,2618 /-0,0001$ & 0,019 & 0,753 & $-0,1510 / 0,1153$ \\
\hline H11 (horas OD) & 0,073 & 0,215 & $-0,1807 / 0,0850$ & $-0,062$ & 0,293 & $-0,1893 / 0,0762$ \\
\hline H12 (horas OD) & 0,086 & 0,146 & $-0,1820 / 0,0837$ & $-0,093$ & 0,116 & $-0,1766 / 0,0893$ \\
\hline Superior & 0,097 & 0,099 & $-0,2059 / 0,0590$ & $-0,117$ & 0,048 & $-0,2246 / 0,0395$ \\
\hline Inferior & 0,084 & 0,156 & $-0,1988 / 0,0664$ & $-0,119$ & 0,044 & $-0,2885 /-0,0290$ \\
\hline Nasal & 0,063 & 0,286 & $-0,1705 / 0,0955$ & $-0,144$ & 0,015 & $-0,3087 /-0,0511$ \\
\hline Temporal & $-0,028$ & 0,634 & $-0,2379 / 0,0254$ & $-0,008$ & 0,896 & $-0,1530 / 0,1132$ \\
\hline Promedio de la CFNR & 0,132 & 0,025 & $-0,1842 / 0,0821$ & $-0,142$ & 0,016 & $-0,2830 /-0,0224$ \\
\hline Espesor global CFNR & $-0,094$ & 0,110 & $-0,2936 /-0,0345$ & 0,071 & 0,229 & $-0,1113 / 0,1549$ \\
\hline $\operatorname{Imax} / \mathrm{Smax}$ & $-0,063$ & 0,288 & $-0,2068 / 0,0581$ & 0,079 & 0,183 & $-0,0481 / 0,2164$ \\
\hline Smax/Imax & 0,009 & 0,876 & $-0,1387 / 0,1277$ & $-0,015$ & 0,796 & $-0,0869 / 0,1769$ \\
\hline Smax/Tavg & 0,101 & 0,089 & $-0,0530 / 0,2116$ & $-0,049$ & 0,405 & $-0,1248 / 0,1415$ \\
\hline Imax/Tavg & 0,072 & 0,224 & $-0,0550 / 0,2097$ & $-0,045$ & 0,450 & $-0,1664 / 0,0997$ \\
\hline Smax/Navg & 0,012 & 0,835 & $-0,1309 / 0,1354$ & 0,052 & 0,383 & $-0,0320 / 0,2317$ \\
\hline Max-Min & 0,016 & 0,792 & $-0,2218 / 0,0424$ & $-0,038$ & 0,524 & $-0,1833 / 0,0823$ \\
\hline SMAX & 0,102 & 0,085 & $-0,1795 / 0,0863$ & $-0,100$ & 0,091 & $-0,2012 / 0,0639$ \\
\hline IMAX & 0,060 & 0,307 & $-0,2096 / 0,0552$ & $-0,072$ & 0,222 & $-0,2484 / 0,0143$ \\
\hline SAVG & 0,110 & 0,064 & $-0,2023 / 0,0627$ & $-0,106$ & 0,072 & $-0,2088 / 0,0560$ \\
\hline IAVG & 0,083 & 0,162 & $-0,2000 / 0,0651$ & $-0,116$ & 0,049 & $-0,2843 /-0,0244$ \\
\hline Espesor medio CFNR & 0,091 & 0,125 & $-0,2315 / 0,0322$ & $-0,137$ & $\mathbf{0 , 0 2 0}$ & $-0,2961 /-0,0373$ \\
\hline Área vert del anillo & $-0,013$ & 0,828 & $-0,2534 / 0,0102$ & 0,044 & 0,460 & $-0,0735 / 0,1931$ \\
\hline Anchura horiz del anillo & 0,077 & 0,194 & $-0,2598 / 0,0033$ & $-0,044$ & 0,457 & $-0,1232 / 0,1444$ \\
\hline Área de disco & 0,009 & 0,877 & $-0,1361 / 0,1315$ & $-0,046$ & 0,440 & $-0,1776 / 0,0895$ \\
\hline Área de excavación & $-0,050$ & 0,403 & $-0,0616 / 0,2046$ & $-0,014$ & 0,810 & $-0,1988 / 0,0676$ \\
\hline Área de anillo & 0,100 & 0,093 & $-0,1880 / 0,0788$ & $-0,040$ & 0,502 & $-0,1026 / 0,1647$ \\
\hline Relación de área E/D & $-0,078$ & 0,187 & $-0,0849 / 0,1821$ & $-0,013$ & 0,830 & $-0,2092 / 0,0568$ \\
\hline Relación horizontal E/D & $-0,081$ & 0,173 & $-0,1059 / 0,1615$ & $-0,015$ & 0,804 & $-0,2037 / 0,0625$ \\
\hline Relación vertical E/D & $-0,054$ & 0,365 & $-0,0731 / 0,1935$ & $-0,031$ & 0,602 & $-0,2057 / 0,0605$ \\
\hline
\end{tabular}

DM: desviación media; DSM: desviación estándar de la media. 


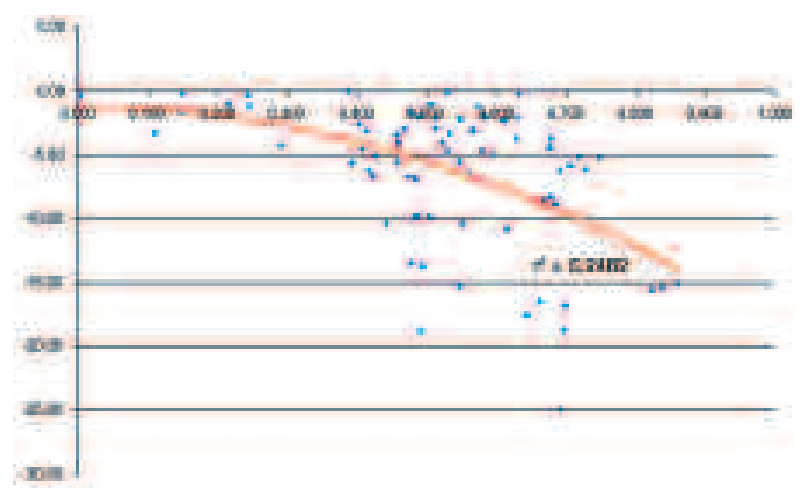

Fig. 1: Recta de regresión entre la desviación media de la perimetría automatizada y la relación del área excavación/disco medida con el HRT II en el grupo de glaucomas. número de parámetros, mostrando un coeficiente de Pearson superior a 0,5 en algunos parámetros: Cuadrante inferior (fig. 2), segmentos horarios del polo inferior, promedio de la capa de fibras nerviosas, el IMAX, el IAVG y el espesor promedio. Las correlaciones fueron débiles o moderadas, y en general, mayores para los parámetros de fibras que para los de la papila

Con respecto al GDx VCC se observaron correlaciones débiles-moderadas (tablas VIII-X) entre la DM y la DSM de la PA y los cinco parámetros de fibras evaluados en el grupo de glaucomas. La correlación más fuerte la presentó la desviación estándar TSNIT con la DM $(0,460)$ (fig. 3) seguida por el NFI también con la DM $(-0,415)$.

Tabla VII. Corelación de los índices principales del campo visual con los parámetros de ibras y papila de la OCT en el grupo de glaucomas

\begin{tabular}{|c|c|c|c|c|c|c|}
\hline Correlaciones de Pearson & $\mathrm{DM}$ & $\mathrm{p}$ & IC $95 \%$ & DSM & $\mathrm{p}$ & IC $95 \%$ \\
\hline H1 (sentido horario OD) & 0,234 & 0,045 & $0,0060 / 0,4392$ & $-0,034$ & 0,775 & $-0,2603 / 0,1962$ \\
\hline H2 (horas OD) & 0,206 & 0,079 & $-0,0240 / 0,4146$ & $-0,044$ & 0,707 & $-0,2702 / 0,1860$ \\
\hline H3 (horas OD) & 0,143 & 0,226 & $-0,0889 / 0,3593$ & $-0,037$ & 0,755 & $-0,2632 / 0,1932$ \\
\hline H4 (horas OD) & 0,144 & 0,222 & $-0,0877 / 0,3604$ & $-0,139$ & 0,238 & $-0,3560 / 0,0926$ \\
\hline H5 (horas OD) & 0,490 & $\mathbf{0 , 0 0 0}$ & $0,2941 / 0,6459$ & $-0,399$ & 0,000 & $-0,5751 /-0,1877$ \\
\hline H6 (horas OD) & 0,544 & 0,000 & $0,3598 / 0,6868$ & $-0,477$ & 0,000 & $-0,6359 /-0,2785$ \\
\hline H7 (horas OD) & $\mathbf{0 , 5 0 4}$ & 0,000 & $0,3115 / 0,6570$ & $-0,446$ & 0,000 & $-0,6120 /-0,2419$ \\
\hline H8 (horas OD) & 0,468 & 0,000 & $0,2687 / 0,6295$ & $-0,294$ & 0,011 & $-0,4900 /-0,0707$ \\
\hline H9 (horas OD) & 0,103 & 0,385 & $-0,1290 / 0,3234$ & 0,108 & 0,360 & $-0,1236 / 0,3283$ \\
\hline H10 (horas OD) & 0,256 & $\mathbf{0 , 0 2 8}$ & $0,0289 / 0,4575$ & $-0,065$ & 0,582 & $-0,2893 / 0,1659$ \\
\hline H11 (horas OD) & 0,408 & 0,000 & $0,1980 / 0,5822$ & $-0,205$ & 0,080 & $-0,4137 / 0,0252$ \\
\hline H12 (horas OD) & 0,243 & $\mathbf{0 , 0 3 7}$ & $0,0151 / 0,4465$ & $-0,078$ & 0,511 & $-0,3008 / 0,1536$ \\
\hline Superior & 0,353 & 0,002 & $0,1355 / 0,5382$ & $-0,129$ & 0,272 & $-0,3476 / 0,1021$ \\
\hline Inferior & $\mathbf{0 , 5 9 2}$ & 0,000 & $0,4199 / 0,7225$ & $-0,511$ & 0,000 & $-0,6624 /-0,3202$ \\
\hline Nasal & 0,208 & 0,075 & $-0,0214 / 0,4168$ & $-0,102$ & 0,387 & $-0,3230 / 0,1295$ \\
\hline Temporal & $\mathbf{0 , 3 7 1}$ & 0,001 & $0,1555 / 0,5525$ & $-0,124$ & 0,292 & $-0,3429 / 0,1075$ \\
\hline Promedio de la CFNR & $\mathbf{0 , 5 3 3}$ & 0,000 & $0,3469 / 0,6789$ & $-0,307$ & 0,008 & $-0,5006 /-0,0847$ \\
\hline Espesor global CFNR & $-0,007$ & 0,953 & $-0,2350 / 0,2220$ & 0,179 & 0,127 & $-0,0514 / 0,3917$ \\
\hline $\operatorname{Imax} / \operatorname{Smax}$ & 0,243 & $\mathbf{0 , 0 3 7}$ & $0,0157 / 0,4469$ & $-0,309$ & 0,007 & $-0,5017 /-0,0861$ \\
\hline Smax/Imax & $-0,265$ & 0,022 & $-0,4654 /-0,0390$ & 0,376 & 0,001 & $0,1610 / 0,5564$ \\
\hline Smax/Tavg & $-0,035$ & 0,769 & $-0,2611 / 0,1953$ & 0,037 & 0,756 & $-0,1934 / 0,2630$ \\
\hline Imax/Tavg & 0,203 & 0,084 & $-0,0272 / 0,4120$ & $-0,326$ & 0,005 & $-0,5160 /-0,1052$ \\
\hline Smax/Navg & 0,017 & 0,886 & $-0,2123 / 0,2446$ & 0,089 & 0,453 & $-0,1428 / 0,3108$ \\
\hline Max-Min & 0,391 & 0,001 & $0,1788 / 0,5689$ & $-0,235$ & 0,044 & $-0,4401 /-0,0071$ \\
\hline SMAX & 0,242 & $\mathbf{0 , 0 3 8}$ & $0,0139 / 0,4455$ & $-0,055$ & 0,641 & $-0,2801 / 0,1756$ \\
\hline IMAX & $\mathbf{0 , 5 2 7}$ & 0,000 & $0,3391 / 0,6741$ & $-0,449$ & 0,000 & $-0,6143 /-0,2455$ \\
\hline SAVG & 0,331 & 0,004 & $0,1105 / 0,5199$ & $-0,127$ & 0,280 & $-0,3457 / 0,1043$ \\
\hline IAVG & 0,565 & 0,000 & $0,3866 / 0,7029$ & $-0,514$ & 0,000 & $-0,6641 /-0,3229$ \\
\hline Espesor medio CFNR & $\mathbf{0 , 5 3 1}$ & $\mathbf{0 , 0 0 0}$ & $0,3439 / 0,6771$ & $-0,339$ & 0,003 & $-0,5269 /-0,1200$ \\
\hline Área vert del anillo & 0,369 & 0,001 & $0,1534 / 0,5510$ & $-0,359$ & 0,002 & $-0,5430 /-0,1422$ \\
\hline Anchura horiz del anillo & 0,443 & 0,000 & $0,2384 / 0,6096$ & $-0,323$ & 0,005 & $-0,5138 /-0,1023$ \\
\hline Área de disco & $-0,001$ & 0,991 & $-0,2298 / 0,2272$ & 0,069 & 0,558 & $-0,1619 / 0,2931$ \\
\hline Área de excavación & $-0,307$ & 0,008 & $-0,5007 /-0,0848$ & 0,324 & 0,005 & $0,1036 / 0,5148$ \\
\hline Área de anillo & $\mathbf{0 , 4 3 2}$ & 0,000 & $0,2255 / 0,6010$ & $-0,385$ & 0,001 & $-0,5638 /-0,1714$ \\
\hline Relación de área E/D & $-0,425$ & 0,000 & $-0,5954 /-0,2173$ & 0,406 & 0,000 & $0,1954 / 0,5804$ \\
\hline Relación horizontal E/D & $-0,333$ & 0,004 & $-0,5222 / 0,1137$ & 0,356 & 0,002 & 0,1391/0,5407 \\
\hline Relación vertical E/D & $-0,487$ & 0,000 & $-0,6438 /-0,2908$ & 0,400 & 0,000 & $0,1884 / 0,5756$ \\
\hline
\end{tabular}

DM: desviación media; DSM: desviación estándar de la media. 
Tabla VIII. Correlación de los índices principales del campo visual con los parámetros del GDx VCC, en el grupo control

\begin{tabular}{lcccccc}
\hline Correlaciones de Pearson & DM & $p$ & IC 95\% & DSM & $p$ & IC 95\% \\
\hline NFI & $-0,017$ & 0,916 & $-0,3154 / 0,2851$ & 0,022 & 0,891 & $-0,2806 / 0,3199$ \\
Promedio TSNIT & $-0,097$ & 0,538 & $-0,3857 / 0,2099$ & 0,104 & 0,508 & $-0,2030 / 0,3919$ \\
Promedio Superior & $-0,151$ & 0,333 & $-0,4320 / 0,1562$ & 0,182 & 0,243 & $-0,1252 / 0,4574$ \\
Promedio Inferior & $-0,041$ & 0,795 & $-0,3370 / 0,2628$ & 0,014 & 0,930 & $-0,2877 / 0,3129$ \\
Desviación estándar TSNIT & $-0,081$ & 0,604 & $-0,3726 / 0,2245$ & 0,063 & 0,686 & $-0,2415 / 0,3570$ \\
\hline \hline
\end{tabular}

DM: desviación media; DSM: desviación estándar de la media.

En general, las correlaciones fueron más fuertes entre la DM y los parámetros estructurales de las tres pruebas que para la DSM.

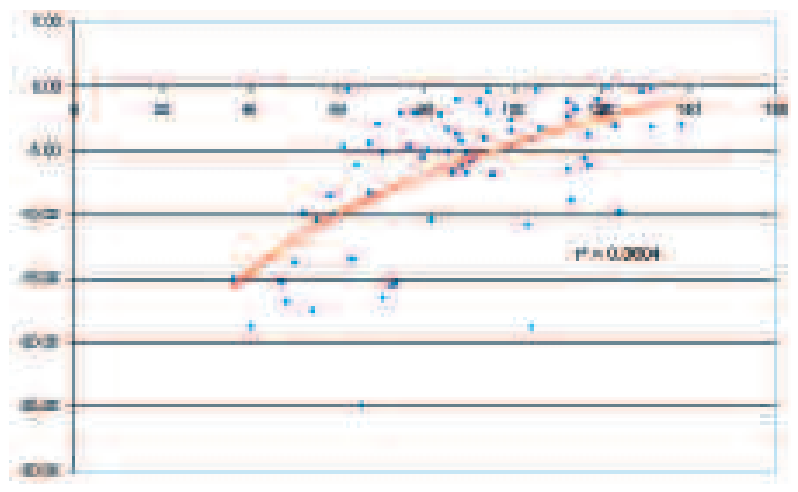

Fig. 2: Recta de regresión entre la desviación media de la perimetría automatizada y el espesor medio de la CFNR en el cuadrante inferior medido con el OCT en el grupo de glaucomas.

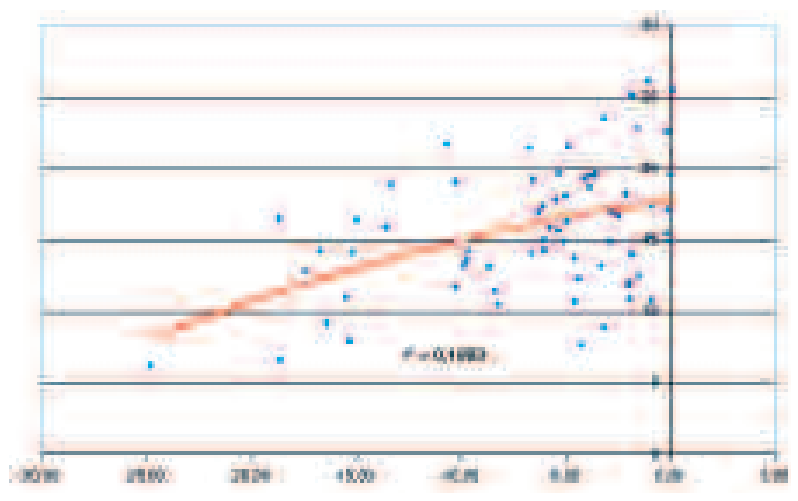

Fig. 3: Recta de regresión entre la desviación media de la perimetría automatizada y la desviación TSNIT medido con el GDx VCC en el grupo de glaucomas.

\section{DISCUSIÓN}

La perimetría automatizada convencional ha sido considerada como la prueba de referencia para el estudio funcional de la enfermedad. La aparición de nuevas técnicas de estudio de la cabeza del nervio óptico y la CFNR de forma objetiva y reproducible, hace interesante contrastar sus resultados con los perimétricos.

Hace ya más de dos décadas que Quigley (9) y asociados demostraron que antes de que se detecte algún cambio en la función visual, se pueden llegar a perder el $40 \%$ de los axones de las células ganglionares. Sommer (10) y colaboradores publicaron que el $60 \%$ de los pacientes hipertensos oculares sufrían alteraciones de la CFNR que precedían en 6 años a la aparición de defectos en la PA, recientemente, el estudio de tratamiento de hipertensos oculares (11) ha demostrado que el $55 \%$ de los ojos que pasan a ser glaucomatosos tenían lesión estructural progresiva del disco óptico sin alteración alguna en la perimetría.

Conviene comenzar la discusión comentando posibles errores. Los sujetos HTO presentaban valores de espesor corneal superiores a los de los otros 2 grupos aunque sin existir diferencias estadísticamente significativas entre ellos. Sin embargo, es posible que el mayor valor paquimétrico sobrevalore las cifras de PIO (12) en este grupo encontrándose ahora sí diferencias entre los HTO y el resto en esta variable al no aplicar ningún factor de corrección de espesor corneal, es decir, se podrían haber clasificado erróneamente como hipertensos a sujetos sanos. De hecho, este grupo presentaba mejores valores de DM y DSM que los normales (sin ser diferencias significativas), no ocurriendo así con los valores de excavación/disco vertical, donde los sujetos con hipertensión ocular mostraron peores valores que los normales aunque de nuevo sin 
Tabla IX. Correlación de los índices principales del campo visual con los parámetros del GDx VCC, en el grupo de hipertensos oculares

\begin{tabular}{lcccccc}
\hline Correlaciones de Pearson & DM & p & IC 95\% & DSM & p \\
\hline NFI & $-0,010$ & 0,869 & $-0,0141 / 0,2748$ & 0,075 & 0,207 & $-0,0375 / 0,2265$ \\
Promedio TSNIT & $-0,064$ & 0,283 & $-0,2776 /-0,0171$ & $-0,008$ & 0,899 & $-0,1502 / 0,1161$ \\
Promedio Superior & 0,014 & 0,808 & $-0,2360 / 0,0274$ & $-0,041$ & 0,486 & $-0,1579 / 0,1084$ \\
Promedio Inferior & $-0,169$ & 0,112 & $-0,2957 /-0,0368$ & 0,040 & 0,497 & $-0,1004 / 0,1657$ \\
Desviación estándar TSNIT & 0,048 & 0,416 & $-0,2093 / 0,0555$ & $-0,038$ & 0,522 & $-0,1602 / 0,1059$ \\
\hline \hline
\end{tabular}

DM: desviación media; DSM: desviación estándar de la media.

Tabla X. Correlación de los índices principales del campo visual con los parámetros del GDx VCC, en el grupo de glaucomas

\begin{tabular}{|c|c|c|c|c|c|c|}
\hline Correlaciones de Pearson & $\mathrm{DM}$ & $\mathrm{p}$ & IC $95 \%$ & DSM & $\mathrm{p}$ & IC $95 \%$ \\
\hline NFI & $-0,415$ & 0,000 & $-0,5875 /-0,2057$ & 0,282 & 0,015 & $-0,0577 / 0,4800$ \\
\hline Promedio TSNIT & 0,276 & 0,017 & $0,0506 / 0,4745$ & $-0,224$ & 0,055 & $-0,4308 / 0,0044$ \\
\hline Promedio Superior & 0,411 & 0,000 & $0,2010 / 0,5843$ & $-0,243$ & 0,037 & $-0,4470 /-0,0157$ \\
\hline Promedio Inferior & 0,308 & 0,008 & $0,0856 / 0,5013$ & $-0,285$ & 0,014 & $-0,4819 /-0,0602$ \\
\hline Desviación estándar TSNIT & 0,460 & 0,000 & $0,2590 / 0,6232$ & $-0,184$ & 0,117 & $-0,3958 / 0,0465$ \\
\hline
\end{tabular}

DM: desviación media; DSM: desviación estándar de la media.

ser estadísticamente significativo. Este hecho no parece deberse al tamaño papilar elevado de HTO que condicione mayores relaciones excavación/disco fisiológicas (como se muestra en los valores de área de disco en la tabla I) ni en principio a una mayor experiencia perimétrica ya que a todos los sujetos del estudio se les realizó el mismo número de perimetrías.

Las correlaciones entre los índices de la PA y los parámetros estructurales de las tres pruebas analizadas, fueron más fuertes y significativas en el grupo de glaucomas. La correlación de Pearson muestra la fuerza de asociación entre dos variables. En el grupo de glaucomas, la variación en la profundidad de los defectos del campo visual para cada uno de los casos permite valorar la concordancia con los cambios en las variables estructurales. Sin embargo, el escaso rango de valores de la DM y la DSM en el grupo de normales e hipertensos condiciona que las correlaciones sean muy débiles o no significativas. Estos dos grupos se comportaron de forma similar ya que desde el punto de vista funcional y anatómico presentan similares características.

No se observaron grandes diferencias en los resultados de las correlaciones para cada uno de los dos índices de la PA, aunque en general la DM presentó las correlaciones más fuertes con las variables estructurales estudiadas.

Las correlaciones más fuertes del HRT se observaron entre la DM de la PA y las relaciones del área excavación/disco y anillo/disco (-0,474 y 0,474 respectivamente). La alteración de estos parámetros nos debe hacer esperar la aparición de defectos glaucomatosos en el campo visual y viceversa.

Las asociaciones más fuertes del OCT con la perimetría se dan en el espesor global de la CFNR y en los espesores del eje vertical, especialmente en el polo inferior, donde habitualmente se distribuyen más fibras, y por tanto es más fácil detectar su pérdida. Fue en el grupo de glaucoma donde el espesor del cuadrante inferior de la CFNR y la DM de la PA presentaron la correlación más fuerte $(0,592)$ de todas las pruebas.

Las correlaciones de los índices de la PA fueron más fuertes con los parámetros que valoran el estado de la CFNR y algo más débiles para los que estudian la morfología papilar, tanto en el OCT como en las otras pruebas morfológicas, posiblemente se deba a que a estos láseres confocales de barrido les resulta más fácil detectar cambios glaucomatosos en la CFNR que en la papila. La amplísima variedad de morfologías papilares hace que establecer el límite entre normalidad y enfermedad en ellas sea difícil, mientras que la distribución de las fibras en la retina es más constante.

Los parámetros papilares del HRT (relación del área de excavación/disco y relación del área de ani1lo/disco, en el grupo de glaucomas) y del OCT (relación excavación/disco vertical, en el grupo de glaucomas) que mejores correlaciones presentaron, 
mostraron una fuerza de asociación bastante similar con los índices de la PA, en concreto $\pm 0,474$ y 0,487 con la DM, respectivamente. El mejor parámetro de la CFNR del OCT (espesor del cuadrante inferior en el grupo de glaucomas) presentó correlaciones discretamente más fuertes con la DM (0,592), mientras que en el caso del GDx VCC (desviación TSNIT en el grupo de glaucomas) las correlaciones fueron algo menores y similares a las primeras citadas $(0,460)$. Las ecuaciones de regresión muestran que la mejor asociación fue para PAOCT.

Otros estudios $(1,13)$ que han evaluado la relación entre HRT y PA, han concluido que las asociaciones con más fuerza se dan entre la DM y el área del anillo seguido por la morfología de la excavación. En nuestro estudio el área del anillo presenta en tercer mejor coeficiente de Pearson en su relación con la DM, y el más alto en el caso de la DSM.

Varios estudios han valorado la relación entre OCT y campo visual. Se ha visto que el espesor en la CFNR es menor en los sectores no glaucomatosos de ojos con glaucoma que en los sectores correspondientes de ojos normales, aunque ello no implique diferencia en cuanto a la función visual (14). En las áreas glaucomatosas de la CFNR correspondientes al defecto del campo visual, el espesor de la misma es menor que en los segmentos no glaucomatosos. Los cambios en el espesor macular detectados con OCT se correlacionan bien con los cambios de la función visual y atrofia de la CFNR (15), sin embargo, las medidas del espesor de la CFNR peripapilar con OCT tienen mayor capacidad de discriminación entre ojos con y sin defectos en el campo visual que las medidas en el área macular (16).

Otros autores $(17,18)$ han evidenciado que en aquellas zonas en las que el espesor de la CFNR o ANR están más adelgazados existe una alteración en el campo visual. Los defectos en hemirretina superior se corresponden con alteraciones del campo visual inferior y viceversa. Los defectos estructurales son más frecuentes y precoces en el polo inferior, seguidos del superior, siendo la zona nasal la que menos se lesiona.

Tarek y colaboradores (19) demuestran que las medidas con OCT del espesor de la CFNR en pacientes glaucomatosos se relacionan en extensión y profundidad con una disminución de la sensibilidad en el campo visual, y las zonas más frecuentemente adelgazadas de la CFNR corresponden a los sectores inferiores. En nuestro estudio también resultó mayor la relación en el cuadrante inferior.

El NFI fue el segundo parámetro que mostró mayor fuerza de asociación en el grupo de glaucomas. Numerosos trabajos $(4,14)$ han evidenciado que el NFI es el parámetro con mejor rendimiento diagnóstico de esta técnica. Nuestros resultados confirman que cuanto mayor es el NFI, más negativa es la DM y mayor es la DSM.

Los estudios transversales tienen limitaciones a la hora de examinar la relación entre estructura y función, ya que pueden ayudar a determinar qué parámetros se asocian con la función visual pero no su relación a lo largo del tiempo. Además, la cantidad de daño en la CFNR necesaria para producir defectos detectables en el campo visual es muy dependiente de cada caso concreto. Por tanto, el desarrollo de estudios longitudinales aportará información sobre cómo se reflejan en el campo visual los daños anatómicos que se van produciendo a lo largo de la enfermedad glaucomatosa.

La diferente naturaleza de los parámetros obtenidos con las pruebas de imagen y la PA hacían esperar correlaciones débiles o moderadas. Así ha sido, pero ello no indica una falta de correspondencia entre ellas, sino que la enfermedad presenta cambios que pueden ser detectados mejor por una prueba o por otra según el caso.

\section{BIBLIOGRAFÍA}

1. Uchida H, Brigatti L, Caprioli J. Detection of structural damage from glaucoma with confocal laser image analysis. Invest Ophthalmol Vis Sci 1996; 37: 2393-2401.

2. Hoh ST, Greenfield DS, Mistlberger A, Liebmann JM, Ishikawa H, Ritch R. Optical coherence tomography and scanning laser polarimetry in normal, ocular hypertensive, and glaucomatous eyes. Am J Ophthalmol 2000; 129: 129-135.

3. Bowd C, Zangwill LM, Berry CC, Blumenthal EZ, Vasile $C$, Sanchez-Galeana $C$, et al. Detecting early glaucoma by assessment of retinal nerve fiber layer thickness and visual function. Invest Ophthalmol Vis Sci 2001; 42: 1993-2003.

4. Medeiros FA, Zangwill LM, Bowd C, Weinreb RN. Comparision of the GDx VCC scanning laser polarimeter, HRT II, confocal scanning laser ophthalmoscope, and stratus OCT optical coherence tomograph for the detection of glaucoma. Arch Ophthalmol 2004; 122: 827-837.

5. Greaney MJ, Hoffman DC, Garway-Heath DF, Nakla M, Coleman AL, Caprioli J. Comparision of optic nerve imaging methods to distinguish normal eyes from those with glaucoma. Invest Ophthalmol Vis Sci 2002; 43: 140-145.

6. Chylack LT Jr, Wolfe JK, Singer DM, Leske MC, Bullimore MA, Bailey IL, et al. The Lens Opacities Classification 
System III. The Longitudinal Study of Cataract Study Group. Arch Ophthalmol 1993;111:831-836.

7. Caprioli J. Automated perimetry in glaucoma. Am J Ophthalmol 1991; 111: 235-239.

8. Wollstein G, Garway-Heath DF, Hitchings RA. Identification of early glaucoma cases with the scanning laser ophthalmoscope. Ophthalmology 1998; 105: 1557-1563.

9. Quigley HA, Addicks EM, Green RW. Optic nerve damage in human glaucoma. III. Quantitative correlation of nerve fiber loss and visual field defect in glaucoma, ischemic neuropathy, papilledema, and toxic neuropathy. Arch Ophthalmol 1982; 100: 135-146.

10. Sommer A, Katz J, Quigley HA, Miller NR, Robin AL, Riehter RG, et al. Clinically detectable nerve fiber atrophy precedes the onset of glaucomatous field loss. Arch Ophthalmol 1991; 109: 77-83.

11. Kass MA, Heuer DK, Higginbotham EJ, Johnson CA, Keltner JL, Miller JP, et al. The Ocular Hypertension Treatment Study: a randomized trial determines that topical ocular hypotensive medication delays or prevents the onset of primary open- angle glaucoma. Arch Ophthalmol 2002; 120: 701-713.

12. Ehlers N, Bramsen T, Sperling S. Appanation tonometry and central corneal thickness. Acta Ophthalmol (Copenh) 1975; 53: 34-43.

13. Iester M, Mikelberg FS, Courtright P, Drance SM. Correlation Between the Visual Field Índices and Heidelberg
Retina Tomograph Parameters. J Glaucoma 1997; 6: 7882.

14. Reus NJ, Lemij HG. Diagnostic accuracy of the GDx VCC for glaucoma. Ophthalmology 2004; 111: 1860-1865.

15. Bagga H, Greenfield DS. Quantitative assessment of structural damage in eyes with localized visual field abnormalities. Am J Ophthalmol 2004; 137: 797- 805.

16. Wollstein G, Schuman JS, Price LL, Aydin A, Beaton SA, Stark PC, et al. Optical coherence tomography (OCT) macular and peripapillary retinal nerve fiber layer measurements and automated visual fields. Am J Ophthalmol 2004; 138: 218-225.

17. Moreno-Montañés J, Álvarez-Vidal A, Sainz-Gómez C, Rodríguez-Conde R. Capa de fibras nerviosas en ojos glaucomatosos. Estudio realizado mediante OCT y su comparación con el campo visual. Arch Soc Esp Oltalmol 2002; 77: 435-441.

18. Anton A, Yamagishi N, Zangwill L, Sample PA, Weinreb $R N$. Mapping structural to functional damage in glaucoma with standard automated perimetry and confocal scanning laser ophthalmoscopy. Am J Ophthalmol 1998; 125: 436446.

19. El Beltagi TA, Bowd C, Boden C, Amini P, Sample PA, Zangwill LM,et al. Retinal nerve fiber layer thickness measured with optical coherence tomography is related to visual function in glaucomatous eyes. Ophthalmology 2003; 110: 2185-2191. 